\title{
Diverse effects of clarithromycin and proposal of its clinical application for treating COVID-19 as a repurposing drug
}

\author{
Takemori, N. ${ }^{1 *}$, Ooi, H.K. ${ }^{2,3}$, Imai, G. ${ }^{4}$, Saio, M. ${ }^{5}$ \\ ${ }^{1}$ Department of Internal Medicine, Division of Hematology, Imai Hospital, Tanaka-cho 100, Ashikaga, Tochigi 326-0822, Japan \\ ${ }^{2}$ Yamazaki University of Animal Health Technology, 4-7-2 Minami-osawa, Hachioji, Tokyo 192-0364, Japan \\ ${ }^{3}$ Laboratory of Parasitology, School of Veterinary Medicine, Azabu University, 1-17-71 Fuchinobe, Sagamihara, \\ Kanagawa 252-5201, Japan \\ ${ }^{4}$ Department of Internal Medicine, Imai Hospital, Tanaka-cho 100, Ashikaga, Tochigi 326-0822, Japan \\ ${ }^{5}$ Laboratory of Histopathology \& Cytopathology, Department of Laboratory Sciences, Gunma University, \\ Graduate School of Health Sciences, 39-22, 3-chome, Showa-machi, Maebashi, Gunma 371-8514, Japan \\ *Corresponding author: takemori_nobuo@circus.ocn.ne.jp
}

\section{ARTICLE HISTORY}

Received: 20 June 2021

Revised: 12 July 2021

Accepted: 12 July 2021

Published: 30 August 2021

\begin{abstract}
Outbreak of SARS-CoV-2 has been declared a pandemic, which is a serious threat to human health. The disease was named coronavirus disease 2019 (COVID-19). Until now, several vaccines and a few drugs have been approved for the prevention and treatment for COVID-19. Recently, the effect of some macrolides including clarithromycin (CAM) on COVID-19 has attracted attention. CAM is known to have diverse effects including immunomodulatory and immunosuppressive effects, autophagy inhibition, steroid sparing effect, reversibility of drug resistance, antineoplastic effect, antiviral effect as well as bacteriostatic/bactericidal effect. Many patients with COVID-19 died due to an overwhelming response of their own immune system characterized by the uncontrolled release of circulating inflammatory cytokines (cytokine release syndrome [CRS]). This CRS plays a major role in progressing pneumonia to acute respiratory distress syndrome (ARDS) in COVID-19 patients. It is noteworthy that CAM can suppress inflammatory cytokines responsible for CRS and also has anti-SARS-CoV-2 effect. Considering the rapidly progressive global disease burden of COVID19 , the application of CAM for treating COVID-19 needs to be urgently evaluated. Recently, an open-labeled non-randomized trial using CAM for treating COVID-19 (ACHIEVE) was initiated in Greece in May, 2020. Its results, though preprint, indicated that CAM treatment of patients with moderate COVID-19 was associated with early clinical improvement and containment of viral load. Thus, treatment with CAM as a single agent or combined with other anti-SARSCoV-2 drugs should be tried for treating COVID-19. In this article, we discussed the significance and usefulness of CAM in treating COVID-19.
\end{abstract}

Keywords: COVID-19; clarithromycin; diverse effect; treatment; SARS-CoV-2.

\section{INTRODUCTION}

In March 2020, the World Health Organization (WHO) declared the new coronavirus (SARS-CoV-2) outbreak a pandemic and named the disease as coronavirus disease 2019 (COVID-19). The current estimate of this global disease burden (Johns Hopkins University, Worldometer) is more than 174 million culminated cases and 3.7 million deaths worldwide as of June 11, 2021. Generally, social distancing and lockdown of cities in epidemic countries have been considered to be the only effective means available to limit the impact of virus transmission. Several vaccines, including $m$-RNA and vector vaccines, are currently being applied worldwide for preventing and limiting the spread of COVID-19. The efficacy of the vaccines has been proven, though the adverse effects still remained to be elucidated. Thus far, several repurposing drugs such as remdesivir, favipiravir, lopinavir.ritonavir, chloroquine (CQ), hydroxychloroquine (HCQ), nafamostat, ciclesonide, dexamethasone, tocilizumab and ivermectin have been tested for treating COVID-19-caused by SARSCoV-2 (Tarighi et al., 2021). No definitive drugs for treating COVID-19 have yet been established after WHO declaration of the pandemic. In May 2020, the Food and Drug Administration (FDA) of USA approved Veklury ${ }^{\oplus}$ (remdesivir) for the treatment of COVID-19 in adult and pediatric patients (12 years and older and weighing at least $40 \mathrm{~kg}$ ) who required hospitalization (FDA, 2020a). Currently, only dexamethasone was demonstrated to have beneficial effects by reducing 
mortality in patients who did not require oxygen and mechanical ventilation (RECOVERY Collaborative Group, 2021b). Baricitinib, an anti-inflammatory drug used for treating rheumatism, that acts as JAK1/JAK2 inhibitor has also been approved in May 2021, for use in COVID-19 patients on condition that it be used together with Remdesivir (FDA, 2020b; Eli Lilly Company, 2020).

Macrolides are bacteriostatic antibiotics having a broad spectrum of activity against many gram-positive and atypical bacterial species that cause respiratory tract infections. Erythromycin (EM, 14-membered macrolide antibiotic) is the first macrolide proven clinically to have efficacy in the treatment of rhinovirus and influenza virus. Thereafter, azithromycin (AZM, 15-membered macrolide antibiotic) and clarithromycin (CAM, 14-membered macrolide antibiotic) were also found to be effective against rhinovirus, respiratory syncytial virus, and influenza virus (Min \& Jang, 2012). Besides the aforementioned respiratory tract viruses, Ebola and Zika virus replication have been reported to be suppressed by AZM (Madrid et al., 2015; Bosseboeuf et al., 2018). SARS-CoV-2 is a single-stranded RNA virus like that of Zika and Ebola viruses. Based on the viral feature and the efficacy for Zika and Ebola viruses, AZM has been empirically used for treating COVID-19.

CAM is a unique macrolide antibiotic which has diverse effects as compared to other macrolides. It includes immunomodulatory and immunosuppressive effects, autophagy inhibition, steroid sparing effect, reversibility of drug resistance, antineoplastic effect, antiviral effect as well as bacteriostatic/bactericidal effect (LeBel, 1993; Culić et al., 2001; Kanoh \& Rubin, 2010; Van Nuffel et al., 2015; Takemori et al., 2020). Thus far, we have investigated the effect of CAM on multiple myeloma. During that study, we noticed that CAM could also be applied for the treatment of COVID-19 (Takemori et al., 2020). It is well known that the antibacterial effect of macrolides, including CAM, is related to their ability to inhibit protein synthesis by binding to the subunit $50 \mathrm{~S}$ of the bacterial ribosome (Poehlsgaard \& Douthwaite, 2002). This protein synthesis inhibition by CAM is also involved in the suppression of coronavirus replication, which means that CAM can also be considered as an antiviral agent. In this article, we focused on the antiviral activity and immunomodulatory effect of CAM, and also emphasized on its usefulness in treating COVID-19.

\section{CAM Therapies for COVID-19 that have been reported to date}

An open-labeled non-randomized clinical trial using CAM for treating COVID-19 as a single agent (i.e., ACHIEVE) started in Greece in May, 2020. Just recently, the results, though preprint, were published in medRxiv (Tsiakos et al., 2020). Almost at the same time, Mansilla et al. (2020) started up the "Macrolides-Clarithromycin Task-Force for the Treatment and Prophylaxis of COVID-19 as a single agent".

\section{The clinical trial (ACHIEVE): CAM monotherapy for COVID-19}

The content of ACHIEVE is briefly summarized as follows.

Ninety COVID-19 patients of moderate severity having respiratory tract dysfunction were administered CAM $500 \mathrm{mg}$ every 12 hours for 7 days. For comparison, 90 other patients of standard-of-care (SOC) propensity score-matched concurrent controls were given AZM plus HCQ. For patients with upper respiratory tract dysfunction, the composite endpoint was designated as: (a) not necessary for hospital readmission or (b) lack of progression into lower respiratory tract dysfunction. For patients with lower respiratory tract dysfunction, it was designated as having at least $50 \%$ decrease in respiratory symptoms score at the end-oftreatment (EOT) without progression into severe respiratory failure (SRF). As a secondary endpoint, the incidence of SRF at the test-of-cure (TOC) was set on day 14. For patients treated with CAM, viral load of SARS-CoV-2, biomarkers, mononuclear cells function, and safety were evaluated. The same parameters were also measured in SOC comparators. The primary endpoint was attained in $86.7 \%$ of patients treated with CAM (95\% Cls; 78.1-92.2\%) and $73.3 \%$ of concurrent SOC comparators (95\% Cls; $63.4-81.4 \%)$. The odds ratio for primary endpoint with CAM treatment in univariate analysis was 2.36 (95\% Cls 1.09-5.08; P: 0.039). Results were confirmed after multivariate stepwise logistic regression analysis (odds ratio 3.30; 95\% Cls 1.10-9.87; P: 0.033). At the TOC visit, the incidence of SRF was $12.2 \%$ ( $n=11 ; 95 \% \mathrm{Cls} 6.9-20.6 \%$ ) among patients treated with CAM (odds ratio for SRF 0.38; $95 \% \mathrm{Cls} 0.17-0.84$ ) versus $26.7 \%$ ( $n=24 ; 95 \% \mathrm{Cls} 18.6-36.6 \%)$ among concurrent SOC comparators ( $P$ : 0.023 ).

CAM use resulted in a decrease of circulating values of C-reactive protein, tumor necrosis factor- $\alpha$ (TNF- $\alpha$ ) and-IL-6; an increase in the ratio of Th1 to Th2 mononuclear responses; as well as suppression of SARS-CoV-2 relative viral load. The single CAM treatment for COVID-19 was found to be significantly superior to the concurrent SOC comparators (AZM plus HCQ). No safety concerns were observed. Patients given CAM within the first five days from the manifestation of clinical sign onset showed better responses. In this trial, it is interesting to note that the viral load of SARS-CoV-2 in the nasopharynx of CAM-treated patients was significantly depressed on day 4 and day 8 after the initiation of CAM treatment. CAM was shown to suppress the constitutive $E$ gene and the specific RdRp gene (for transcription of RdRp). CAM treatment also elicits early clinical improvement in patients with moderate COVID-19. Modulation of the Th1/Th2 responses has been proposed as a mechanism to attenuate the hyper-inflammatory reaction of the host, leading to the prevention of cytokine release syndrome (CRS). The results of the ACHIEVE trial clearly show that CAM treatment in patients with moderate COVID-19 is associated with early clinical improvement and containment of viral load. This trial is the first study to show the anti-viral and anti-inflammatory impact of CAM on COVID-19.

\section{Macrolide-CAM Task-Force for the treatment and prophylaxis of COVID-19 as a single agent}

Mansilla et al. (2020) in Argentina started the project in May, 2020, "Macrolides-CAM Task-Force for the treatment and prophylaxis of COVID-19 as a single agent". After extensively reviewing the literature on macrolides for treating COVID-19 and assessing the general mechanism of action of macrolides, they suggested that CAM is not only an antibacterial agent but also has a strong immunomodulatory, anti-inflammatory and anti-viral mode of action. According to Mansilla et al. (2020) CAM treatment as a single agent for COVID-19 would be much simpler, safer and cheaper than giving $\mathrm{CQ}$ or $\mathrm{HCQ}$ alone or in combination with AZM. Furthermore, since the drug could be immediately available in the world, CAM treatment can reduce the number of patients with severe symptoms who need to be hospitalized in the intensive care units, especially if used as soon as the first symptoms appear or even at the confirmation of infection in asymptomatic patients. It would help to reduce the high mortality rate related with the disease. The Macrolide-CAM Task Force now welcomes all interested parties throughout the world to join in. 
Clinical case studies which proved the efficacy of CAM for COVID-19

The number of clinical case studies which demonstrated the efficacy of CAM for COVID-19 is very low. These studies were combination treatments consisting of CAM and other drugs. Sawai et al. (2020) reported three cases of COVID-19 patients with interstitial pneumonia. These patients were successfully treated with oral CAM (200mg twice a day, for 1 week) combined with ciclesonide (Alvesco ${ }^{\circ}$ ) (inhalation of $800 \mu \mathrm{g}$, once a day, for 2 weeks) and oral favipiravir (Avigan ${ }^{\circ}$ ) (1800 mg, twice a day on day 1 , subsequently followed by 800 mg, twice a day for day 2-14). However, no clinical trials consisting of CAM, ciclesonide and favipiravir have been tried later. In Colombia, Millán-Oñate et al. (2020) reported the recovery of a 34-year-old Columbian patient with COVID19 pneumonia after being given $C Q$ and intravenous administration of CAM. Excellent outcomes were also reported in Ecuador for 12 patients, who were treated with a combination of CAM, n-acetylcysteine and an antiviral nutraceutical (Viusid) (Ojeda Crespo et al., 2020). These case studies seem to indicate that CAM might be a potent candidate drug worth evaluating for the treatment of COVID-19.

\section{Mechanism of Inhibitive Actions of CAM on SARS-CoV-2}

\section{1) The direct inhibition of CAM on SARS-CoV-2 replication}

It should be noted that the mode of replication of SARS-CoV2 is unique because it represents a positive-sense singlestranded RNA virus. Its replication process seems somewhat complicated as shown in Figure 1. After the entry of SARSCoV-2 via angiotensin-converting enzyme 2 (ACE2) receptor into the host cytoplasm, the release of viral g-RNA occurs. Subsequently, open reading frame (ORF) $1 \mathrm{a}$ and ORF1b are translated to produce polyproteins $1 \mathrm{a}$ (pp1a) and pp1ab, with the assistance of host ribosomes. These polyproteins are cleaved into 16 mature nonstructural proteins (nsp1nsp16) by viral proteases. The nsp12 represents RNAdependent RNA polymerase (RdRp). Viral genomic replication and subgenomic (structural) transcription (transcription for nucleocapsid, spike, membrane and envelope) occur within the replication-transcription complex (RTC) using RdRp. Translation of viral structural proteins (i.e., spike, membrane and envelope proteins) takes place at the endoplasmic reticulum (ER) membrane. Then, the nucleocapsid (i.e., replicated viral genome and nucleocapsid protein) combines with structural proteins (i.e., spike, envelope and membrane structural proteins) in the Golgi complex. Finally, mature virions formed inside Golgi vesicles are released via exocytosis to the outside of the cell (Alanagreh et al., 2020).

Macrolides including CAM, EM and AZM can bind to the 23S ribosomal RNA ( $r R N A$ ) component of the $50 \mathrm{~S}$ ribosome and interfere with the assembly of $50 \mathrm{~S}$ subunits. These macrolides prevent elongation of polypeptide chain at the transpeptidation step of synthesis by blocking the $50 \mathrm{~S}$ polypeptide export tunnel (Sigma-Aldrich, 2006). Elongation of polypeptide chain is prematurely terminated after a small peptide has been formed but cannot move past the macrolide roadblock (Sigma-Aldrich, 2006; Sohmen et al., 2009). This will lead to the inhibition of peptide chain elongation and hinder the production of RdRp, resulting in the inhibition of SARSCoV-2 replication. This mechanism seems to be an important process for the viral containment.

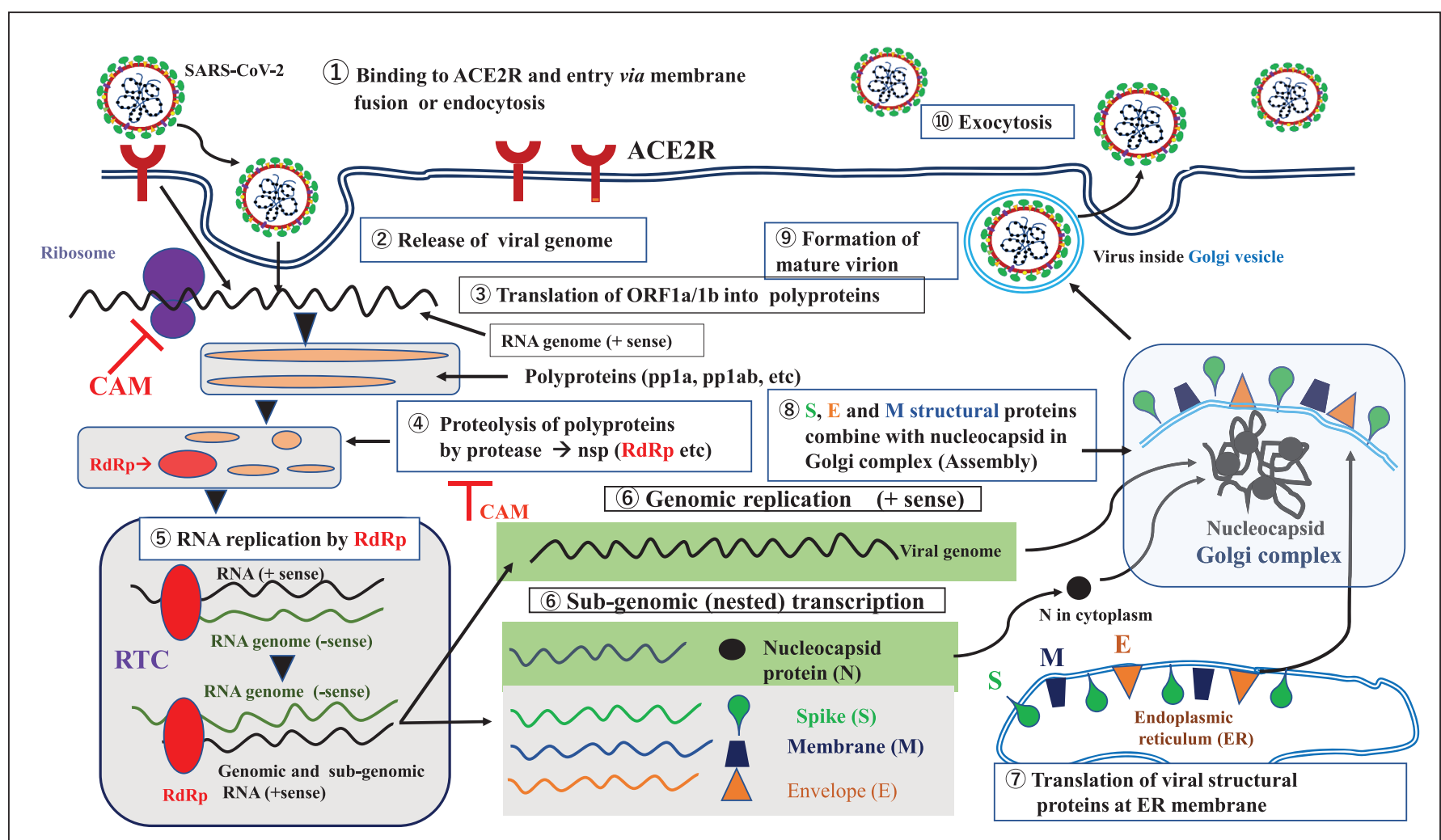

Figure 1. Entry, replication and maturation process of SARS-CoV-2. Abbreviations: ACE2R, angiotensin-converting enzyme 2 receptor; ORF, open reading frame; RTC, replication-transcription complex; RdRp, RNA-dependent RNA-polymerase; nsp, non-structural protein. [A modified schema, cited from Alanagreh et al., 2020]. 


\section{2) Structure-based drug selection}

Anti-viral drugs include inhibitors against viral protease, integrase and polymerase enzymes. These inhibitors appear to be effective in terms of blocking virus replication and may prove to be a promising treatment for COVID-19. Recently, Dayer (2020) performed docking experiments using COVID-19 EC 3,4,2 protease with 305 amino acid residues which is comparable to SARS protease with 305 residues. They selected drugs from 9 HIV-1 protease inhibitors and 21 candidates from anti-bronchitis drugs based on their chemical structures as ligands. The binding capacity and the inhibitory potency of the candidate drugs are as follows: Tipranavir $>$ indinavir $>$ atazanavir $>$ darunavir $>$ ritonavir $>$ amprenavir for HIV-1 protease inhibitors and cefditoren $>$ cefixime $>E M>C A M$ for anti- bronchitis medicines. Based on the above data, CAM, though less potent, seems to have anti-SARS-CoV-2 effect by blocking the viral protease (Figure 1).

\section{3) Increased in pH of trans-Golgi network by CAM may lead to inhibition of SARS-COV-2 binding to glycosylated ACE2 receptor}

Poschet et al. (2020) found that in primary cystic fibrosis (CF), the bronchial epithelial cells of CF treated with AZM led to an increase in the $\mathrm{pH}$ of trans-Golgi network from $6.1 \pm 0.2$ to $6.7 \pm 0.1$ at a concentration of $100 \mu \mathrm{M}$ for 1 hour, or $1 \mu \mathrm{M}$ for 48 hours. Treatment of the same cells with AZM $100 \mu \mathrm{M}$ for 1 hour increased the $\mathrm{pH}$ of the recycling endosome from 6.1+0.1 to $6.7+0.2$. Both the trans-Golgi network and recycling endosome are known to play important roles in the packaging of proteins into vesicles that are destined for secretion. In COVID-19 infection, this packaging process is important to facilitate viral replication and spread. Altering the $\mathrm{pH}$ of these organelles may interfere with these intracellular viral activities. In addition, the raised $\mathrm{pH}$ of the trans-Golgi network may alter glycosylation of ACE2 receptor. Alteration of glycosylation of the receptor may lead to the inhibition of SARS-CoV-2 binding to the host cells (Gbinigie \& Frie, 2020). Being quite similar to AZM, CAM is known to increase $\mathrm{pH}$ in endosome and reduce nuclear factor- $\mathrm{KB}$ (NF-KB) protein in seasonal influenza (INF) infection. Yamaya et al. (2010) reported that CAM can reduce the expression of sialic acids with an $\alpha 2,6$ linkage (SA $\alpha, 26 \mathrm{Gal})$ partly through the inhibition of nuclear factor- $\kappa B(N F-\kappa B)$, and increasing the $\mathrm{pH}$ in endosome of the airway epithelial cells. Thus, CAM might also interfere with these intracellular viral activities of SARS-CoV-2 through these mechanisms.

\section{4) Induction of secretory-IgA (S-IgA) class-switching recombination (CSR) by CAM}

The dimeric $\operatorname{Ig} A$ is called secretory $\lg A(S-\lg A)$, which is a polymer consisting of two IgA monomers, a J chain and a glycoprotein called the secretory component (SC). It represents the main immunoglobulin found in tears, saliva, sweat, colostrum and secretions from the gastrointestinal tract, genito-urinary tract, prostate and respiratory epithelium. S-IgA is considered an important first line of defense against many invading pathogens including viruses. Shinahara et al. (2013) treated 195 INF-A virus infected children with oseltamivir (OSV) and zanamivir (ZNV) with or without CAM. They observed that the combination of CAM plus OSV or ZNV boosted and restored the production of antigenspecific mucosal IgA and systemic IgG. Takahashi et al. (2012) examined the effects of CAM on S-IgA immune responses by using INF-A virus (H1N1)-infected mice treated with or without OSV. They demonstrated that CAM enhanced S-IgA production and neutralizing activities through the induction of IgA CSR and upregulation of the expression of B-cell activating factor of TNF family (BAFF) molecules in mucosal dendritic cells in INF-A virus infected mice. Thus, the possibility of antiviral effects of CAM through antigen-specific S-IgA via CSR was suggested. It is probable that the same effect of CAM will occur in COVID-19 infection.

\section{5) Immunomodulatory and immunosuppressive effects}

Data accumulated to date suggested that many COVID-19 patients died due to an excessive response of their immune system. This has been characterized by the abnormal release of circulating cytokines including IL-1 $\beta$, IL-6, IL-12, IL-18, TNF- $\alpha$, TGF- $\beta$, GM-CSF, interferon- $\gamma($ IFN- $\gamma$ ) and various chemokines (e.g, IL-8, monocyte chemotactic factor [MCP-1] and IFN- $\gamma$ inducible protein [IP-10]) (Mangalmurti \& Hunter, 2020; Moor \& June, 2020; Sun et al., 2020; Tang et al., 2020). This phenomenon is referred to as CRS or cytokine storm (Mangalmurti \& Hunter, 2020; Moor \& June, 2020; Sun et al., 2020; Tang et al., 2020). In particular, a crucial role seems to be played by IL- 6 , TNF- $\alpha$ and IL-1. CRS has been attributed as a major factor in the deterioration of COVID-19 patients with pneumonia into acute respiratory distress syndrome (ARDS), that cumulates in systemic inflammation and multiorgan failure (Takahashi et al., 2012). CAM is also known to be effective for treating organizing pneumonia (Pathak et al., 2014). Cai et al. (2013) investigated whether alveolar macrophages produce aberrant pro-inflammatory cytokines in bronchiolitis obliterans organizing pneumonia (BOOP) and whether this can be inhibited by CAM or AZM. They demonstrated that spontaneous and lipopolysaccharidestimulated alveolar macrophages from patients with BOOP showed an increase in the production of TNF- $\alpha$, soluble TNF receptor 1 (TNFR1) and TNFR2, IL-1 $\beta$, IL-6, IL-8, IL-10, IFN- $\gamma$-inducible protein (IP-10) and CC chemokine ligand 18 (CCL18). CAM and AZM induced a dose-dependent suppression of spontaneous TNF- $\alpha$, STNFR2, IL-6, IL- 8 and CCL18 production. CAM also inhibited the IL-1 $\beta$ production. CAM and AZM significantly and dose-dependently attenuated the LPS-stimulated production of STNFR1, STNFR2, IL- 8 and CCL18. CAM also inhibited the LPS-stimulated TNF- $\alpha, I L-1 \beta$, IL-6 and IL-10 production. Čulić et al. (2001) reported that CAM can inhibit IL-1, IL-6, IL- 8 , TNF- $\alpha$, TGF- $\beta$ and RANTES (regulated upon activation, normal T-cell expressed and secreted). CAM has been reported to suppress almost all cytokines and chemokines involved in CRS. Considering its pleiotropic immuno-modulatory and anti-inflammatory effects, CAM might play a very important role in the treatment of COVID-19. It should be noted that CAM represents a potent inhibitor of IL-6, TNF- $\alpha$ and IL-1, which are the three major cytokines responsible for inflammation (Takemori et al., 2020).

\section{6) Anti-mast cell effect of CAM through inhibiting degranulation of MAST cell granules}

Recently, Kazama et al. reported that anti-allergic drugs (olopatadine, ketotifen), CAM and corticosteroids (hydrocortisone, dexamethasone) can inhibit the degranulation of mast cell granules, and this plays an important role in stabilizing the mast cells (Kazama et al., 2016; Kazama, 2020). Besides T-lymphocytes and macrophages, recent studies additionally indicated a large contribution of mast cells to the pathogenesis of cytokine storm triggered by SARS-CoV- 2 . Once activated by the SARS-CoV-2, mast cells that reside in the respiratory mucous membrane produce and secrete proinflammatory cytokines, such as IL-1, IL-4, IL-5, IL-6, TNF- $\alpha$ in addition to their exocytotic release of chemokines (Kazama et al., 2016). Several studies revealed that mast cells directly facilitate the progression of pulmonary fibrosis by the 


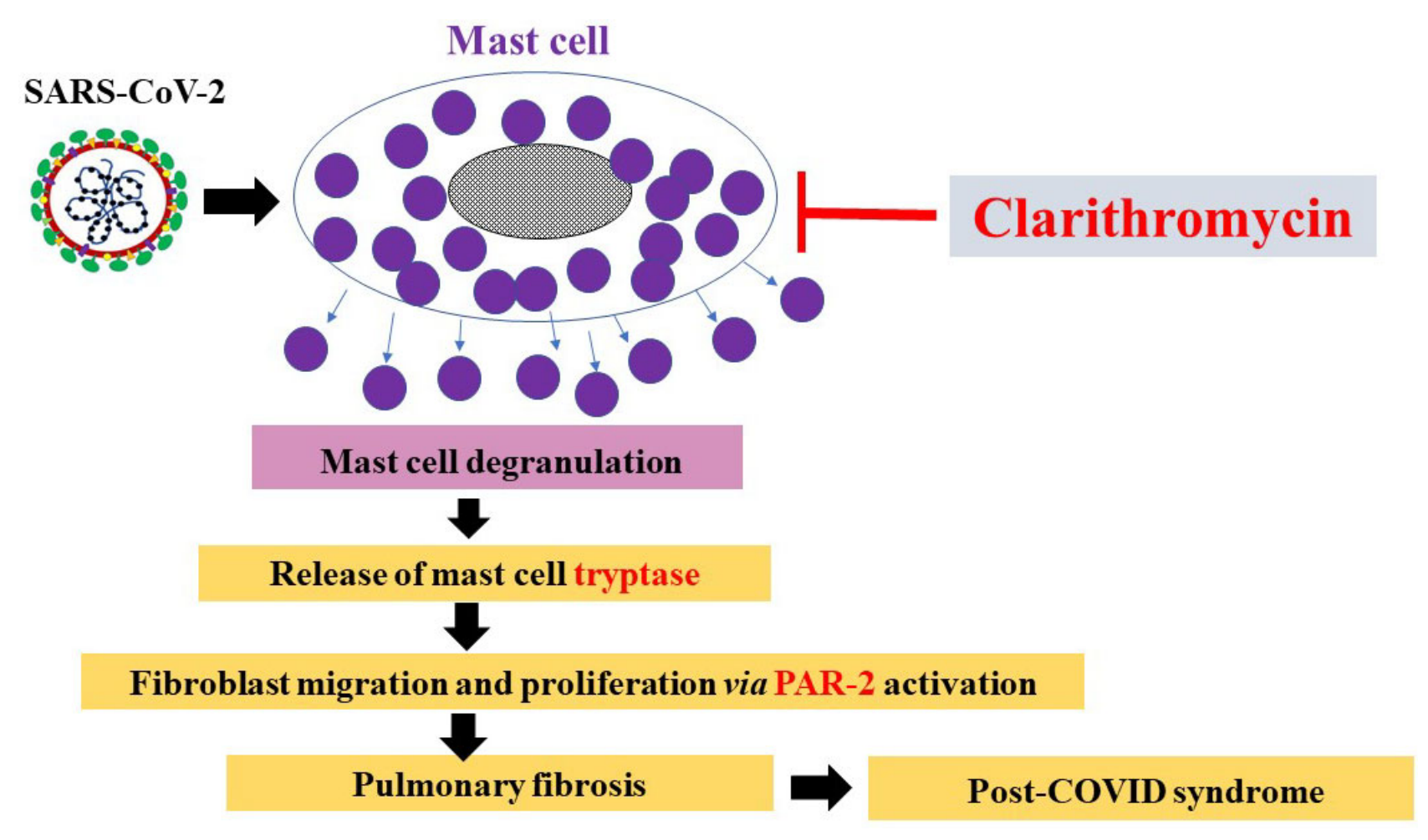

Figure 2. Mast cells in the development of pulmonary fibrosis and inhibitory effect of CAM on mast cell degranulation. SARS-CoV2 infection causes mast cell degranulation and releases mast cell tryptase contained in the granules. Tryptase activates PAR2 , and causes fibroblast migration and proliferation, leading to development of fibrosis and post-COVID syndrome. CAM inhibits degranulation of mast cells.

Abbreviation: PAR-2, protease activated receptor-2. (A modified schema, cited from Kazama, 2020).

exocytotic release of mast cell granules which contain mast cell mediated-tryptase. This tryptase is a trypsin-like serine protease and plays the role of a potent mitogen as a fibroblast growth factor (FGF) (Ruoss et al., 1991; Bagher et al., 2018). In addition, fibroblasts are known to express protease activated receptor 2 (PAR2) that may be activated by tryptase (Bagher et al., 2018) (Figure 2).

Regardless of the severity of COVID-19, a high proportion of the patients had to struggle with persistent respiratory or systemic symptoms after recovery. This is called "post-COVID syndrome" in which pulmonary fibrosis is one of the pathogenic manifestations. COVID-19 pneumonia is an interstitial pulmonary pneumonia characterized by the proliferation of fibroblasts, excessive deposition of collagen and extracellular matrix. Mast cells directly facilitate the progression of pulmonary fibrosis via exocytic release of tryptase. It should be noted that CAM has an ability to potently inhibit the degranulation of mast cell.

\section{Recommended Agents Combined with CAM}

\section{1) Ciclesonide (Alvesco $\left.{ }^{\circledR}\right)$}

Matsuyama et al. (2021) examined 92 steroid compounds chosen from the Prestwick Chemical Library to assess their inhibitory effects on the cytopathological changes induced by betacoronaviruses, which include MERS-CoV and SARSCoV-2. They assayed the test compounds in SARS-CoV-2 infected VeroE6/TMPRSS2 cells, by quantifying the viral RNA using in real-time PCR. Moreover, they also assayed the viral load in Vero cells treated with steroid compounds followed by infection with MERS-CoV at a multiplicity of infection (MOI) of 0.1 and then incubated for 3 days. Of the 92 steroid compounds examined, ciclesonide exhibited not only low cytotoxicity but also potent suppression of the replication of MERS-CoV as well as SARS-CoV-2. To date, no inhalable steroid other than ciclesonide has been found to have antiviral effect against COVID-19. Generally, steroid treatment for COVID-19 is not recommended due to the possibility of prolonged viremia and complications such as diabetes, but systemic administrations of hydrocortisone, methylprednisolone, dexamethasone and prednisolone are still being considered (Iwabuchi et al., 2020).

Ciclesonide is an inhalant prodrug that stays on the lung surface with only little diffusion into the blood vessels. Clinically, ciclesonide is used as an inhalant for treating asthma. Recently, its possible efficacy for COVID-19 was suggested at the "Emergency Expansion Meeting on New Coronavirus Infections" held on February 19, 2020. The suppressive effect of ciclesonide on viral replication is specific to coronaviruses, highlighting it as a candidate drug for the treatment of COVID-19. Based on this suggestion, Iwabuchi et al. (2021) treated three patients having mild to moderate COVID-19 with ciclesonide and obtained favorable results. The mechanism by which ciclesonide plays an important role in improving COVID-19 pneumonia is thought to be its anti-PAK-1 effect. According to Salvi (2020), PAK-1 (RAC/CDC42-activated kinase 1 ) is a major pathogenic serinethreonine kinase that is widely associated with a variety of diseases such as cancers, inflammation, viral infection, immunosuppression and aging. PAK-1 is known to be activated by different viruses, including SARS-CoV-2. PAK-1 not only enhances viral replication, but also releases key 


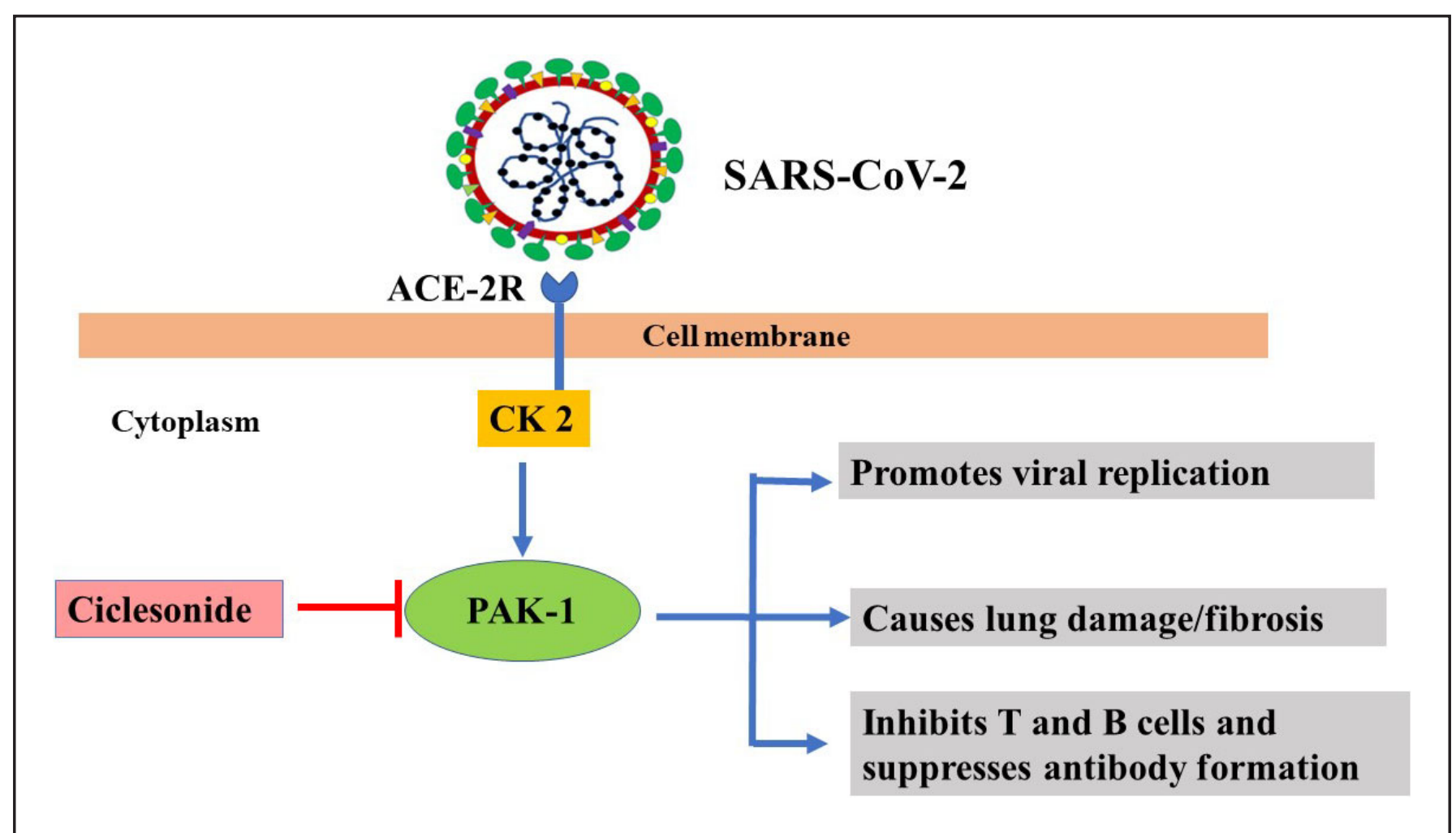

Figure 3. Possible mechanisms of action of ciclesonide in COVID-19 through inhibiting PAK-1. Abbreviations: CK-2, casein kinase 2; PAK-1, RAC/CDC42-activated kinase-1 (A modified schema cited from Salvi, 2021).

inflammatory mediators that cause lung damage, and it switches off the cell-mediated immunity so that antiviral antibody production is inhibited (Figure 3). PAK-1 inhibitors have therefore been suggested to have a potent role in the management of COVID-19. Some of the well-known PAK-1 inhibitors include propolis, melatonin, $\mathrm{HCQ}$, ivermectin, and ketorolac. Ciclesonide has recently been shown to possess PAK-1 inhibiting properties (Maruta \& He, 2020). Thus, the effects exerted by ciclesonide might be through its inhibitory effect on PAK-1.

In Japan, ciclesonide has frequently been combined with favipiravir for treating COVID-19. However, the evaluation of its therapeutic result still remains in the realm of discussion. Based on this scientific evidence, the combination treatment consisting of CAM and ciclesonide for patients with slight respiratory symptoms might be effective.

\section{2) Favipiravir (Avigan ${ }^{\circledR}$ )}

Favipiravir is an antiviral drug used to treat influenza in Japan. It is, however, only indicated for novel influenza (strains that cause more severe disease) rather than seasonal influenza. There are evidences that its use during pregnancy may result in harm to the baby. Teratogenic and embryotoxic effects had been shown experimentally (Jin et al., 2013; Hayden \& Shindo, 2019). The mechanism of its antiviral action is thought to be related to the selective inhibition of viral RdRp (Jin et al., 2013; Hayden \& Shindo, 2019). Favipiravir is a prodrug that is metabolized to its active form, favipiravir-ribofranosyl-5'-triphosphate which functions as a nucleotide analogue to selectively inhibit RdRp of the influenza virus. It mimics both guanosine and adenosine (Jin et al., 2013). Incorporating two such bases in a row stops primer extension leading to a blockage of RNA synthesis. A prospective open-label multicenter trial involving adult patients with COVID-19 was conducted from February to March, 2020 in China. In that trial, favipiravir was compared with umifenovir (Arbidol, used as an antiviral drug to treat influenza in Russia) with respect to the time to relief for pyrexia, cough and other secondary outcomes. It was observed that favipiravir did not significantly improve the clinical recovery rate. However, favipiravir significantly shortened the duration to relief for pyrexia and coughing (Chen et al., 2020). In Japan, favipiravir has not been authorized as a treatment for COVID-19. Nevertheless, a combination treatment consisting of CAM, ciclesonide and favipiravir for patients with severe respiratory syndrome such as ARDS might be effective. In fact, Sawai et al. succeeded in treating COVID-19 interstitial pneumonia with CAM, ciclesonide and favipiravir (Sawai et al., 2020).

\section{3) Hydroxychloroquine (HCQ)/chloroquine (CQ) plus azithromycin (AZM)}

$\mathrm{HCQ}$ and $\mathrm{CQ}$ are 4-aminochloroquine drugs developed in the mid-20 th century for the treatment of malaria (Ben-Zvi et al., 2012). HCQ is considered to be a class equivalent to CQ. Both drugs have been used in the treatment of autoimmune diseases because of their immunomodulatory effects on several cytokines, including IL-4 and IL-6. There are some evidences that these drugs also have antiviral properties against many different viruses, including coronavirus (BenZvi et al., 2012). In addition, AZM, widely used as an antibacterial agent, has also been shown to possess in vitro antiviral activity against a variety of ribonucleic acid viruses (Gielen et al., 2010; Dyall et al., 2014; Li et al., 2019).

Based on this evidence, in March 2020, Gautret et al. (2020) first conducted an open-labeled non-randomized clinical trial applying HCQ and AZM as a treatment of COVID19. They reported that despite its small sample size, HCQ treatment was significantly associated with viral load 
reduction/disappearance in COVID-19 patients and this effect was reinforced by AZM. However, a study from another French group failed to replicate the favorable results of the clinical trial that was carried out in Marseille, France (Molina et al., 2020). From 13 April 2020 to 1 August 2020, the Qatar Prospective RCT of Expediting Coronavirus Tapering (QPROTECT) was carried out to assess the cure rate of HCQ with or without AZM in cases of low-acuity COVID-19. However, the Q-PROTECT findings showed no favorable effect of HCQ with or without AZM in mild or asymptomatic patients with SARS-CoV-2 infection (Omrani et al., 2020). The authors concluded that there was insufficient evidence to support HCQ as a useful drug for COVID-19. In this connection, Cavalcanti et al. (2020) conducted a multicenter, randomized, open-label, three-group (HCQ, HCQ plus AZM and SOC) controlled clinical trial (COALITION COVID-19 Brazil Investigators) in hospitalized patients with mild-to-moderate COVID-19. Among these hospitalized patients, the use of HCQ alone or $\mathrm{HCQ}$ with AZM did not improve their clinical status as compared with SOC. The same group of investigators performed an open-labeled, randomized clinical trial (COALITION II trial) at 57 centers in Brazil (Furtado et al., 2020). Patients were allocated to treatments with AZM plus SOC (a regimen that included $H C Q)(n=214)$ or SOC without AZM $(n=183)$. The results showed that adding AZM to SOC did not result in clinical improvement or mortality reduction in patients admitted to hospital with severe COVID-19. Both COALITION trials only showed evidence for the safety of AZM.

The available guidelines (FDA) suggests that AZM should not be used in combination with HCQ outside of the context of clinical trials, due to the lack of high-quality evidence in favor and concerns about their potential adverse effects (FDA, 2020b; Singh et al., 2021) Furthermore, RECOVERY (Randomized Evaluation of COVID-19 Therapy carried out from April to December, 2020) assessed the AZM monotherapy effect against patients admitted to hospital with COVID-19 (RECOVERY Collaborative Group, 2021a). The results showed that AZM did not improve the survival or other prespecified clinical outcomes. RECOVERY Collaborative Group concluded that AZM use in patients admitted to hospital with COVID-19 should be restricted to those in whom there is a need for clear antimicrobial indication. The IDSA (infectious disease society of America) guideline panel recommended $\mathrm{HCQ} / \mathrm{CQ}$ plus AZM only in the context of a clinical trial (Bhimraj et al., 2020). Nevertheless, in some countries, the treatments for COVID-19 with AZM combined with $\mathrm{HCQ}$ or $\mathrm{CQ}$ have been continued. Until now, no large scale clinical trials using CAM combined with $\mathrm{HCQ}$ or $\mathrm{CQ}$ have been done. Only clinical case report using CAM and CQ in treating COVID-19 has been documented thus far (Mansilla et al., 2020). Further large scale meticulous clinical trials should be expected.

\section{Comparison of CAM versus AZM in the Treatment of COVID-19}

Zimmermann et al. (2018) reviewed the immunomodulatory effects of macrolides including CAM and AZM. The most frequently reported outcomes of the macrolides were a decrease in the number of neutrophils, and the increased concentration of neutrophil elastase, IL- $1 \alpha$, IL- 6 , IL-8, TNF- $\beta$, eosinophil cationic protein, and matrix metalloproteinase 9. A decrease in T helper 2 (Th2) cell cytokines (eg, IL-4, IL-5, IL-6) was reported more frequently than that of Th1 cytokines $(e g, \mathrm{IL}-2, \mathrm{IFN}-\gamma)$. Basic patterns of the effects of CAM and AZM on immunological markers seem to be almost similar (LeBel, 1993). In contrast to AZM, CAM has diverse effects (LeBel, 1993; Čulić, 2001; Kanoh \& Rubin, 2010; Van Nuffel et al., 2015; Takemori et al., 2020) and is more effective in suppressing cytokine production than AZM (Pathak et al., 2014). AZM is pharmacologically very similar to CAM, but, according to our clinical experience and scientific literature, CAM as an antiviral, anti-inflammatory and/or immunomodulatory drug seems to be much better (Mansilla et al., 2020). AZM has longer half-lives and unique penetration as compared to CAM. Generally, AZM is used for short-course-administration (500 $\mathrm{mg}$ on day 1 , followed by $250 \mathrm{mg} /$ day on day 2 through 5). On the other hand, CAM is usually used for 7-14 day course (250 mg twice daily for 7-14 days). Considering that the COVID-19 runs protracted clinical course, the short-course administration of AZM seems somewhat inappropriate for controlling this disease, whereas CAM seems more suitable than AZM.

\section{Possible Effectiveness of CAM on SARS-CoV-2 Variants}

SARS-CoV-2 is a positive-sense single-stranded RNA virus. This means that frequent replication errors will occur during the infection process because of the insufficient RNA repair system pertinent to the positive-sense single-stranded RNA viruses. Considering the antiviral and immunomodulatory effects of CAM, it seems likely that CAM will also be effective for the variant types of COVID-19.

\section{Adverse effects of CAM}

The common adverse effects of CAM are diarrhea, nausea, abnormal taste, dyspepsia, abdominal pain/discomfort, headache, insomnia, tooth discoloration, smell loss and taste loss, liver dysfunction, somnolence, confusion, allergic reactions ranging from urticaria and mild skin eruptions to anaphylaxis, Steven-Johnson syndrome and toxic epidermal necrosis, (LeBel, 1993; Čulić, 2001; Kanoh \& Rubin, 2010; FDA, 2015; Van Nuffel et al., 2015; Takemori et al., 2020). Most of these adverse effects are usually mild in intensity and resolve after the discontinuation of treatment. In our experience, though rare in frequency, severe liver dysfunction (drug-induced hepatitis caused by CAM) occurred within 1 week after the initiation of CAM administration. Thus, regular liver function tests of once a week should be done at least for one month. If any dysfunctional parameter is detected, CAM administration should be discontinued immediately. Furthermore, administration of CAM is also known to prolong QT interval in the ECG. Rarely, it can lead to fatal ventricular arrhythmias, including ventricular tachycardia and torsades de pointes (Owens Jr \& Nolin, 2006). Thus, CAM therapy is not recommended for patients with a history of arrhythmia and QT prolongation. Since CYP3A4 is inhibited by CAM, coadministration of CAM with drugs metabolized by CYP3A4 should be done carefully (Takemori et al., 2020). In our experience, we have used CAM 200-400 mg, twice a day for 3-4 weeks for treating patients with multiple myeloma, Hodgkin's lymphoma and sarcoidosis (Takemori et al., 2020). No severe adverse effects have been observed thus far except for a rare occurrence of drug-induced hepatitis.

\section{Economical Merits, Widespread Availability of CAM}

Widespread availability of generic versions and the low price of CAM made it an attractive option for COVID-19 treatment as it could reduce the cost of treatment. CAM treatment as a single agent could be much more simple, safer and cheaper than giving $\mathrm{CQ}$ or $\mathrm{HCQ}$ alone or in combination with AZM as well as other therapeutic options. It would help to reduce the high mortality rate associated with the disease and enhance the inactivation of the viral load probably regardless of the virus variant types. 


\section{CONCLUSION}

In this article, the antiviral effect of CAM on SARS-CoV-2 and its immunomodulatory effect in COVID-19 treatment were discussed and emphasized. The recent ACHIEVE clinical trial (Tsiakos et al., 2020) has demonstrated that CAM monotherapy in patients with moderate COVID-19 can achieve early clinical improvement and reduction of viral load. This trial is the first systematic report demonstrating the effectiveness and usefulness of CAM in treating COVID-19. Based on the pharmacological and immunological mechanisms of CAM, treatment with CAM alone or CAM in combination with other drugs such as ciclesonide and favipiravir might be promising and open the possibility of an international strategy in the fight against COVID-19.

\section{Competing interests}

All authors declare no conflict of interests.

\section{Abbreviations}

ACE2: Angiotensin-converting enzyme2; ARDS: Acute respiratory distress syndrome; AZM: Azithromycin; BAFF: Bcell activating factor of TNF family; BOOP: Bronchiolitis obliterans organizing pneumonia; CAM: Clarithromycin; CCL18: Chemokine (c-c motif) ligand 18; CF: Cystic fibrosis; $\mathbf{C l}$ : Confidential interval; CK 2: Casein kinase 2; COVID-19: Corona virus disease 2019; CQ: Chloroquine; CRS: Cytokine release syndrome; CSR: Class-switch recombination; CYP3A4: Cytochrome P450 3A4; ECG: Electrocardiogram; EM: Erythromycin; EOT: End-of-treatment; ER: Endoplasmic reticulum; FDA: Food and Drug Administration; FGF: Fibroblast growth factor; HCQ: Hydroxychloroquine; HIV: Human immunodeficiency virus; IFN: Interferon; IL: Interleukin; INF: Influenza; IP-10: Interferon- $\gamma$ inducible protein (CXCL10); JAK: Janus kinase; MCP-1: Monocyte chemotactic protein-1; MOI: Multiplicity of infection; NF-KB: Nuclear factor-KB; nsp: Nonstructural protein; OSV: Oseltamivir; ORF: Open reading frame; PAK-1: RAC/CDC42-activated kinase 1; PAR-2: Protease activated receptor 2; pp: Polyprotein; RANTES: Regulated upon activation, normal T-cell expressed and secreted; RCT: Randomized controlled trial; RdRp: RNA-dependent RNApolymerase; rRNA: Ribosomal RNA; RTC: Replicationtranscription complex; SARS: Severe acute respiratory syndrome; SARS-CoV-2: Severe acute respiratory syndrome coronavirus 2; SOC: Standard of care; SRF: Severe respiratory failure; TGF- $\beta$ : Transforming growth factor- $\beta$; Th2: T helper 2; TMPRRSS2: Transmembrane protease, serine 2; TNF- $\alpha$ : Tumor necrosis factor- $\alpha$; TOC: Test-of-cure; WHO: World Health Organization; ZNV: Zanamivir.

\section{REFERENCES}

Alanagreh, L., Alzoughool, F. \& Atoum, M. (2020). The human coronavirus disease COVID-19: its origin, characteristics, and insights into potential drugs and its mechanisms. Pathogens 9: 331. https://doi.org/10.3390/pathogens 9050331

Bagher, M., Larsson-Callerfelt, A-K., Rosmark, O., Hallgren, O., Bjermer, L. \& Westergren-Thorsson, G. (2018). Mast cells and mast cell tryptase enhance migration of human lung fibroblasts through protease-activated receptor 2. Cell Communication and Signaling 16: 59. https://doi.org/10.1186/ s12964-018-0269-3

Ben-Zvi, I., Kivity, S., Langevitz, P. \& Shoenfeld, Y. (2012). Hydroxychloroquine: From malaria to autoimmunity. Clinical Reviews in Allergy and Immunology 42: 145-153. https://doi.org/10.1007/s12016-010-8243-x
Bhimraj, A., Morgan, R.L., Shumaker, A.H., Lavergne, V., Baden, L., Chen, V.C-C., Edwards, K.M., Gandhi, R., Muller, W.J. \& O'horo, J.C. (2020). Infectious Diseases Society of America Guidelines on the treatment and management of patients with coronavirus disease 2019 (COVID-19). Clinical Infectious Diseases ciaa478. https://doi.org/10.1093/cid/ciaa478

Bosseboeuf, E., Aubry, M., Nhan, T., de Pina, J.J., Rolain, J.M., Raoult, D. \& Musso, D. (2018). Azithromycin inhibits the replication of Zika virus. Journal of Antivirals and Antiretrovirals, 10: 6-11. https://doi.org/10.4172/19485964.1000173

Cai, M., Bonella, F., Dai, H., Sarria, R., Guzman, J. \& Costabel, U. (2013). Macrolides inhibit cytokine production by alveolar macrophages in bronchiolitis obliterans organizing pneumonia. Immunobiology 218: 930-937. https://doi.org/10.1016/j.imbio.2012.10.014

Cavalcanti, A.B., Zampieri, F.G., Rosa, R.G., Azevedo, L.C.P., Veiga, V.C., Avezum, A., Damiani, L.P., Marcadenti, A., Kawano-Dourado, L. \& Lisboa, T. (2020). Hydroxychroloquine with or without azithromycin in mild-tomoderate Covid-19. New England Journal of Medicine 383: 2041-2052. https://doi.org/10.1056/NEJMoa2019014

Chen, C., Zhang, Y., Huang, J., Yin, P., Cheng, Z., Wu, J., Chen, S., Zhang, Y., Chen, B., Lu, M. et al. (2020). Favipiravir versus arbidol for COVID-19: A randomized clinical trial. medR $\chi$ iv [Preprint] https://doi.org/10.1101/2020.03.17.20037432

Čulić, O., Eraković, V. \& Parnham, M.J. (2001). Anti-inflammatory effects of macrolide antibiotics. European Journal of Pharmacology 429: 209-229. https://doi.org/10.1016/S00142999(01)01321-8

Dayer, M.R. (2020). Old drugs for newly emerging viral disease, COVID-19: Bioinformatic prospective. Ar xiv: 2003.04524 [q-bio.BM]. [Preprint]

Dyall, J., Coleman, C.M., Hart, B.J., Venkataraman, T., Holbrook, M.R., Kindrachuk, J., Johnson, R.F., Olinger Jr., G.G., Jahrling, P.B., Laidlaw, M. et al. (2014). Repurposing of clinically developed drugs for treatment of middle-east respiratory syndrome coronavirus infection. Antimicrobial Agents and Chemotherapy 58: 4885-4893. https://doi.org/10.1128/ AAC.03036-14

Eli Lilly and Company. (2020). News Release. Baricitinib in combination with remdesivir reduces time to recovery in hospitalized patients with COVID-19 in NIAID-sponsored ACTT-2 trial. http://www.prnewswire.com/news-release/ baricitinib-in-combination-with-remdesivir-reducestime-to-recovery-in-hospitalized-patients-with-covid-19in-niaid-sponsored-actt-2-trial-301129865. html

FDA. (2015) BIAXIN. FDA Reference ID: 3836666 https:// www.accessdata.fda.gov/drugsatfda_docs/label/2015/ 050662s057,050698s037,050775s025lbl.pdf

FDA review of safety issues. (2020a). FDA cautions against use of hydroxychloroquine or chloroquine for COVID-19 outside of the hospital setting or a clinical trial due to risk of heart rhythm problems. FDA Reference ID: 4610984 https://www.accessdata.fda.gov/drugsatfda_docs/nda/ 2020/OSE\%20Review_Hydroxychloroquine-Cholorquine \%20-\%2019May2020_Redacted.pdf

FDA News Release. (2020b). COVID-19 Update: FDA broadens emergency use authorization for veklury (remdesivir) to include all hospitalized patients for treatment of COVID-19. https://www.fda.gov/news-events/pressannouncements/covid-19-update-fda-broadensemergency-use-authorization-veklury-remdesivirinclude-all-hospitalized

FDA News Release. (2020c). Coronavirus (COVID-19) Update: FDA authorizes drug combination for treatment of COVID19. https://www.fda.gov/news-events/press-announce 
ments/coronavirus-covid-19-update-fda-authorizes-drugcombination-treatment-covid-19

Furtado, R.H.M., Berwanger, O., Fonseca, H.A., Corrêa, T.D., Ferraz, L.R., Lapa, M.G., Zampieri, F.G., Veiga, V.C., Azevedo, L.C.P., Rosa, R.G. et al. (2020). Azithromycin in addition to standard of care versus standard of care alone in the treatment of patients admitted to the hospital with severe COVID-19 in Brazil (COALITION II): a randomized clinical trial. Lancet 396: 959-967. https://doi.org/10.1016/S01406736(20)31862-6

Gautret, P., Lagier, J-C., Parola, P., Hoang, V.T., Meddeb, L., Mailhe, M., Doudier, B., Courjon, J., Giordanengo, V., Vieira, V.E. et al. (2020). Hydroxychloroquine and azithromycin as a treatment of COVID-19: results of an open-label non-randomized clinical trial. International Journal of Antimicrobial Agents 56: 105949. https://doi.org/10.1016/ j.ijantimicag.2020.105949

Gbinigie, K. \& Frie, K. (2020). What is the evidence for using macrolide antibiotics to treat COVID-19? The Centre for Evidence-Based Medicine (CEBM) 2020. https://www.cebm. net/covid-19/what-is-the-evidence-for-use-of-macrolideantibiotics-for-treatment-of-covid-19/

Gielen, V., Johnston, S.L. \& Edwards, M.R. (2010). Azithromycin induces anti-viral responses in bronchial epithelial cells. European Respiratory Journal 36: 646-654. https://doi.org/ 10.1183/09031936.00095809

Hayden, F.G. \& Shindo, N. (2019). Influenza virus polymerase inhibitors in clinical development. Current Opinion in Infectious Disease 32: 176-186. https://doi.org/10.1097/ QCO.0000000000000532

Iwabuchi, K., Yoshie, K., Kurakami, Y., Takahashi, K., Kato, Y. \& Morishima, T. (2020). Therapeutic potential of ciclesonide inhalation for COVID-19 pneumonia: Report of three cases. Journal of Infection and Chemotherapy 26: 625-632. https:// doi.org/10.1016/j.jiac.2020.04.007

Jin, Z., Smith, L.K., Rajwanshi, V.K., Kim, B. \& Deval, J. (2013). The ambiguous base-pairing and high substrate efficiency of T-705(favipiravir) ribofuranosyl 5'-triphosfate towards influenza A virus polymerase. PLoS One 8: e68347. https://doi.org/10.1371/journal.pone.0068347

Kanoh, S. \& Rubin, B.K. (2010). Mechanisms of action and clinical application of macrolides as immunomodulatory medications. Clinical Microbiology Reviews 23: 590-615. https://doi.org/10.1128/CMR.00078-09

Kazama, I., Saito, K., Baba, A., Mori, T., Abe, N., Endo, Y., Toyama, H., Ejima, Y., Matsubara, M. \& Yamauchi, M. (2016). Clarithromycin dose-dependently stabilizes rat peritoneal mast cells. Chemotherapy 61: 295-303. https://doi.org/ $10.1159 / 000445023$

Kazama, I. (2020). Stabilizing mast cells by commonly used drugs: a novel therapeutic target to relieve post-COVID syndrome? Drug Discoveries and Therapeutics 14: 259-261. https://doi.org/10.5582/ddt.2020.03095

LeBel, M. (1993). Pharmacokinetic properties of clarithromycin: a comparison with erythromycin and azithromycin. Canadian Journal of Infectious Diseases and Medical Microbiology 4: 148-152. https://doi.org/10.1155/1993/168061

Li, C., Zu, S., Deng, Y-Q., Li, D., Parvatiyar, K., Quanquin, N., Shang, J., Sun, N., Su, J., Liu, Z. et al. (2019). Azithromycin protects against Zika virus infection by upregulating virusinduced type I and III interferon responses. Antimicrobial Agents and Chemotherapy 63: e00394-19. https://doi.org/ 10.1128/AAC.00394-19
Madrid, P.B., Panchal, R.G., Warren, T.K., Schurtleff, A.C., Endsley, A.N., Green, C.E., Kolokoltsov, A., Davey, R., Manger, I.D., Gilfillan, L. et al. (2015). Evaluation of Ebola virus inhibitors for drug repurposing. ACS Infectious Diseases 10: 317-326. https://doi.org/10.1021/acsinfecdis.5b00030

Mangalmurti, N. \& Hunter, C.A. (2020). Cytokine storms: understanding COVID-19. Immunity 53: 19-25. https://doi. org/10.1016/j.immuni.2020.06.017

Mansilla, E., Martínez, R.R., Marín, G.H., Filho, I.Z., Rivas, E., Rivas, J., de Carvalho, K.A.T., Dayer, M.R. \& Samadikuchaksaraei, A. (2020). Macrolide-clarithromycin task-force for the treatment and prophylaxis of COVID-19 as a single agent. Pharmacology and Pharmacy 11: 85-104. https://doi.org/10.4236/pp.2020.116009

Maruta, H. \& He, H. (2020). PAK1-blockers: potential therapeutics against COVID-19. Medicine in Drug Discovery 6: 100039. https://doi.org/10.1016/j.medidd.2020.100039

Matsuyama, S., Kawase, M., Nao, N., Shirato, K., Ujike, M., Kamitani, W., Shimojima, M. \& Fukushi, S. (2021). The inhaled steroid ciclesonide blocks SARS-CoV-2 RNA replication by targeting the viral replication-transcription complex in cultured cells. Journal of Virology 95: e0164820. https://doi.org/10.1128/JVI.01648-20

Millán-Oñate, J., Millan, W., Mendoza, L.A., Sánchez, C.G., Fernandez-Suarez, H., Bonilla-Aldana, D.K. \& RodriguezMorales, A.J. (2020). Successful recovery of COVID-19 pneumonia in a patient from Colombia after receiving chloroquine and clarithromycin. Annals of Clinical Microbiology and Antimicrobials 19: 16. https://doi.org/10.1186/ s12941-020-00358-y

Min, J-Y. \& Jang, Y.J. (2012). Macrolide therapy in respiratory viral infections. Mediators of Inflammation 2012: 649570. https://doi.org/10.1155/2012/649570

Molina, J.M., Delaugerre, C., Goff, J.L., Mela-Lima, B., Ponscarme, D., Goldwirt, L. \& de Castro, N. (2020). No evidence of rapid antiviral clearance or clinical benefit with the combination of hydroxychloroquine and azithromycin in patients with severe COVID-19 infection. Medecine et Maladies Infectieuses 50: 384 https://doi.org/ 10.1016/j.medmal.2020.03.006

Moor, J.B. \& June, C.H. (2020). Cytokine release syndrome in severe COVID-19. Science 368: 473-474. https://doi.org/ 10.1126/science.abb8925

Ojeda Crespo, A.O., Ojeda Cedillo, A.J., Ojeda Cedillo, P.O., Ojeda Cedillo, A.E. \& León, A. (2020). New alternative for treatment for COVID 19 in Ecuador. Interamerican Journal of Medicine and Health 3: 1-10. https://doi.org/10.31005/ iajmh.v3i0.82

Omrani, A.S., Pathan, S.A., Thomas, S.A., Harris, T.R.E., Coyle, P.V., Thomas, C.E., Qureshi, I., Bhutta, Z.A., Mawlawi, N.A., Kahlout, R.A. et al. (2020). Randomized double-blinded placebo-controlled trial of hydroxychloroquine with or without azithromycin for virologic cure of non-severe Covid-19. EClinicalMedicine 29-30: 100645. https://doi.org/ 10.1016/j.eclinm.2020.100645

Owens Jr., R.C. \& Nolin, T.D. (2006). Antimicrobial-associated QT interval prolongation: pointes of interest. Clinical Infectious Diseases 43: 1603-1611. https://doi.org/10.1086/ 508873

Pathak, V., Kuhn, J.M., Durham, C., Funkhouser, W.K. \& Henke, D.C. (2014). Macrolide use leads to clinical and radiological improvement in patients with cryptogenic organizing pneumonia. Annals of the American Thoracic Society 11: 87-91. https://doi.org/10.1513/AnnalsATS. 201308-261CR 
Poschet, J.F., Perkett, E.A., Timmins, G.S. \& Deretic, V. (2020). Azithromycin and ciprofloxacin have a chloroquine-like effect on respiratory epithelial cells. BioR iv $^{\text {[Preprint] }}$ https://doi.org/10.1101/2020.03.29.008631

Poehlsgaard, J. \& Douthwaite, S. (2002). The macrolide binding site on the bacterial ribosome. Current Drug Targets Infectious Disorders 2: 67-78. https://doi.org/10.2174/ 1568005024605927

RECOVERY Collaborative Group. (2021a). Azithromycin in patients admitted to hospital with COVID-19 (RECOVERY): a randomized, controlled, open-label, platform trial. Lancet 397: 605-612. https://doi.org/10.1016/S0140-6736(21) 00149-5

RECOVERY Collaborative Group. (2021b). Dexamethasone in hospitalized patients with Covid-19. New England Journal of Medicine 384: 693-704. https://doi.org/10.1056/ NEJMoa2021436

Ruoss, S.J., Hartmann, T. \& Caughey, G.H. (1991). Mast cell tryptase is a mitogen for cultured fibroblasts. Journal of Clinical Investigation 88: 493-499. https://doi.org/10.1172/ $\mathrm{JCl} 115330$

Salvi, S.S. (2021). Is there a role for inhaled ciclesonide in the treatment of COVID-19? Lung India 38: 1-4. https:// doi.org/10.4103/lungindia.lungindia_473_20

Sawai, T., Morio, R., Harada, Y., Yoshioka, S., Matsuo, N., Kadota, J., Izumikawa, K. \& Mukae, H. (2020). Three cases of patients with COVID-19 pneumonia who were successfully treated with favipiravir, ciclesonide and clarithromycin (in Japanese). Case reports of COVID-19 collected and documented by Japanese Respiratory Society in 2020. https://www.jrs.or.jp/uploads/uploads/ files/covid19/30_20200611.pdf

Shinahara, W., Takahashi, E., Sawabuchi, T., Arai, M., Hirotsu, N., Takasaki, Y., Shindo, S., Shibao, K., Yokoyama, T., Nishikawa, K. et al. (2013). Immunomodulator clarithromycin enhances mucosal and systemic immune responses and reduces re-infection rate in pediatric patients with influenza treated with antiviral Neuraminidase inhibitors: a retrospective analysis. PLoS One 8: e70060. https://doi. org/10.1371/journal.pone.0070060

Sigma-Aldrich. (2006). Inhibition of protein synthesis by antibiotics. BioFiles 2006: 1.4,17. https://www.labcoltd. com/catalogs? Fpdf $=18$

Singh, H., Chauhan, P. \& Kakkar, A.K. (2021). Hydroxychloroquine for the treatment and prophylaxis of COVID-19: the journey so far and the road ahead. European Journal of Pharmacology 890: 173717. https://doi.org/10.1016/j.ejphar.2020.173717

Sohmen, D., Harms, J.M., Schlünzen, F. \& Wilson, D.N. (2009). SnapShot: Antibiotic inhibition of protein synthesis I. Cell 138: 1248.e1. https://doi.org/10.1016/j.cell.2009.08.001
Sun, X., Wang, T., Cai, D., Hu, Z., Chen, J., Liao, H., Zhi, L., Wei, H., Zhang, Z., Qiu, Y. et al. (2020). Cytokine storm intervention in the early stages of COVID-19 pneumonia. Cytokine and Growth Factor Reviews 53: 38-42. https:// doi.org/10.1016/j.cytogfr.2020.04.002

Takahashi, E., Kataoka, K., Indalao, I.L., Konoha, K., Fujii, K., Chida, J., Mizuno, D., Fujihashi, K. \& Kido, H. (2012). Oral clarithromycin enhances airway immunoglobulin A (IgA) immunity through induction of IgA class switching recombination and B-cell-activating factor of the tumor necrosis factor family molecule on mucosal dendritic cells in mice infected with influenza A virus. Journal of Virology 86: 10924-10934. https://doi.org/10.1128/JVI.01207-12

Takemori, N., Ooi, H-K., Imai, G., Hoshino, K. \& Saio, M. (2020). Possible mechanisms of action of clarithromycin and its clinical application as a repurposing drug for treating multiple myeloma. Ecancermedicalscience 14: 1088. https://doi.org/10.3332/ecancer.2020.1088

Tang, Y., Liu, J., Zhang, D. \& Xu, Z. (2020). Cytokine storm in COVID-19: the current evidence and treatment strategies. Frontiers in Immunology 11: 1708. https://doi.org/10.3389/ fimmu.2020.01708

Tarighi, P., Eftekhari, S., Chizari, M., Sabernavaei, M., Jafari, D. \& Mirzabeigi, P. (2021). A review of potential suggested drugs for coronavirus disease (COVID-19) treatment. European Journal of Pharmacology 895: 173890. https:// doi.org/10.1016/j.ejphar.2021.173890

Tsiakos, K., Tsakiris, A., Tsibris, G., Voutsinas, P., Panagopoulos, P., Kosmidou, M., Petrakis, V., Gravvani, A., Gkavogianni, T., Klouras, E. et al. (2020). Oral clarithromycin in COVID-19 of moderate severity: the ACHIEVE open-label trial using concurrent matched comparators. MedR Xiv [Preprint] https://doi.org/10.1101/2020.12.22.20248753

Van Nuffel, A.M.T., Sukhatme, V., Pantziarka, P., Meheus, L., Sukhatme V.P. \& Bouche, G. (2015). Repurposing drugs in oncology (ReDO)-clarithromycin as an anti-cancer agent. Ecancermedicalscience 9: 513. https://doi.org/10.3332/ ecancer.2015.513

Yamaya, M., Shinya, K., Hatachi, Y., Kubo, H., Asada, M., Yasuda, H., Nishimura, H. \& Nagatomi, R. (2010). Clarithromycin inhibits type A seasonal influenza virus infection in human airway epithelial cells. The Journal of Pharmacology and Experimental Therapeutics 333: 81-90. https://doi.org/ 10.1124/jpet.109.162149

Zimmermann, P., Ziesenitz, V.C., Curtis, N. \& Ritz, N. (2018). The immunomodulatory effects of macrolides a systematic review of the underlying mechanisms. Frontiers in Immunology 9: 302. https://doi.org/10.3389/ fimmu.2018.00302 